\title{
Mecánica sagital del cambio de dirección en jugadoras de fútbol de diferente nivel competitivo
}

\author{
Sagittal mechanics of change of direction in female football \\ players of different competitive levels
}

Violeta García-Quiles ${ }^{1,2}$

Alba Aparicio-Sarmiento ${ }^{2}$

1,2. Facultad de Ciencias del Deporte de San Javier. Campus de Excelencia Internacional de la Universidad de Murcia "Campus Mare Nostrum". 2. Grupo de Investigación Aparato Locomotor y Deporte. Facultad de Ciencias del Deporte de San Javier. Universidad de Murcia.

\begin{abstract}
Resumen
La evaluación del cambio de dirección podría contribuir de forma importante tanto a orientar el entrenamiento preventivo como a guiar los procesos de recuperación de las futbolistas. Los objetivos de este estudio fueron describir la mecánica sagital del cambio de dirección (COD) de un grupo de jugadoras de fútbol y comparar la biomecánica sagital del COD, así como el rendimiento de las jugadoras en la prueba en función de su nivel competitivo. Para ello, se analizaron las mecánicas de flexión de rodilla y flexión de tronco de las futbolistas durante el penúltimo (PA) y último apoyo en un COD de $90^{\circ}$. Se realizó un estudio observacional, descriptivo y de corte transversal con 34 jugadoras de entre 16-28 años pertenecientes a dos equipos femeninos de diferente nivel competitivo. Para el análisis de la biomecánica sagital del COD se utilizó el software Kinovea. El tiempo de ejecución fue calculado mediante células fotoeléctricas. Se encontró que la posición media del tronco fue en flexión en el momento del contacto inicial durante el penúltimo apoyo, tanto en el COD hacia la derecha $\left(7,0 \pm 4,8^{\circ}\right)$, como en el COD hacia la izquierda $\left(6,1 \pm 5,6^{\circ}\right)$. La flexión de rodilla máxima (DER: $\left.94,1 \pm 21^{\circ} ; \mathrm{IZQ}: 91,5 \pm 26,7^{\circ}\right)$ fue considerablemente superior (valores inferiores)

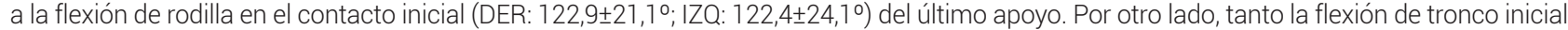
como máxima durante el último apoyo, presentaron valores significativamente superiores en el equipo de futbolistas de élite (inicial: 12,1 $\pm 6,7^{\circ}$ vs. 5,2 $\pm 6,3^{\circ}$; máxima: $13,4 \pm 6,8^{\circ}$ vs. $7,3 \pm 6^{\circ}$ ). En conclusión, las jugadoras de fútbol mostraron una estrategia de flexión de tronco de bajo riesgo durante el apoyo final, sin embargo, no realizaron una estrategia de deceleración clara y eficiente durante el PA, aunque sí que presentaron una adecuada flexión de rodilla. Las futbolistas de élite realizaron el cambio de dirección más rápido y de forma más segura.
\end{abstract}

Palabras clave: Lesiones, ligamento cruzado anterior, factores de riesgo, mecánica de side-step, paso lateral, flexión de rodilla, cinemática del tronco, maniobras de deceleración.

\begin{abstract}
The assessment of change of direction mechanics could make an important contribution to both targeting preventive functional training and guiding the recovery processes of female players. The purpose of this study was to describe sagittal change of direction (COD) mechanics of a group of female football players and to compare the sagittal biomechanics of the COD as well as the players' performance in the test by their competitive level. Knee flexion and trunk flexion mechanics of the players were analysed during the penultimate and final contact in a $90^{\circ} \mathrm{COD}$. A descriptive cross-sectional study was carried out with 34 players aged 16-28 years belonging to two female teams of different competitive levels. For the analysis of sagittal mechanics, Kinovea software was used and the execution time was recorded using photoelectric time cells. It was found that the average position of the trunk was in flexion at initial contact during the penultimate support, both to the right $\left(7 \pm 4.8^{\circ}\right)$ and to the left $\left(6.1 \pm 5.6^{\circ}\right)$ and it was also found that maximum knee flexion (DER: $94.1 \pm 21^{\circ} ;$ IZQ: $91.5 \pm 26.7^{\circ}$ ) was considerably higher (lower values) than knee flexion at initial contact (DER: $122.9 \pm 21.1^{\circ}$; IZQ: $122.4 \pm 24.1^{\circ}$ ). On the other hand, both initial and maximum trunk flexion during the final contact presented significantly higher values in the elite football team (initial: $12.1 \pm 6.7^{\circ} \mathrm{vs} .5 .2 \pm 6.3^{\circ}$; maximum: $13.4 \pm 6.8^{\circ} \mathrm{vs}$. $7.3^{\circ} \pm 6^{\circ}$ ). In conclusion, female football players showed a low-risk trunk flexion strategy during the final stance, however, they did not perform a clear and efficient deceleration strategy during the penultimate contact, although they did show adequate knee flexion. Elite female footballers performed the change of direction faster and more safely.
\end{abstract}

Keywords: Injuries, anterior cruciate ligament, injury risk factors, cutting mechanics, sidestep, knee flexion, trunk kinematics, deceleration manoeuvres.

*Autor de correspondencia: Alba Aparicio Sarmiento; alba.aparicio@um.es

Recibido: 24 de junio de 2021

Aceptado: 28 de julio de 2021

Publicado: 01 de diciembre de 2021

Como citar (APA): García-Quiles, V., \& Aparicio-Sarmiento, A. (2021). Efecto agudo del programa Knäkontroll sobre parámetros del rendimiento físico en jugadores de fútbol de categoría juvenil. JUMP, (4), 45-58. https://doi.org/10.17561/jump.n4.5

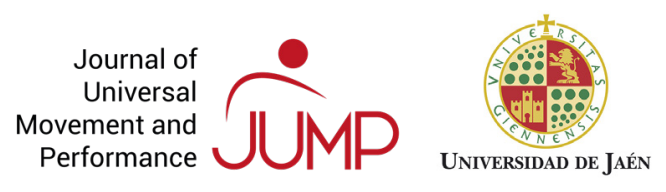




\section{Introducción}

El fútbol femenino ha experimentado un crecimiento exponencial en los últimos años y sigue creciendo en popularidad, con cada vez más niñas y mujeres jugando en todo el mundo (Cardoso de Araújo y Mießen, 2017; Federation Internationale de Football Association, 2019). De hecho, el número de jugadoras federadas se ha quintuplicado desde 1985 a 2014 (UEFA, 2015), llegando a superar actualmente los 13 millones de mujeres jugando al fútbol organizado en todo el mundo, tanto a nivel amateur como de élite (Cardoso de Araújo y Mießen, 2017; Federation Internationale de Football Association, 2019). Según un informe reciente de la Real Federación Española de Fútbol, el número de jugadoras federadas en España alcanzó la cifra de 41898 en la temporada 2018/2019.

Esta reciente y rápida progresión del grado de profesionalización en el fútbol femenino ha llevado a un aumento sustancial de la frecuencia, la intensidad y la competitividad tanto de los entrenamientos como de los partidos, dando lugar a un incremento en el riesgo de lesión (Cardoso de Araújo \& Mießen, 2017; Bradley \& Scott, 2019; Valenti et al., 2018). En este sentido, las lesiones deportivas producen un impacto negativo sobre el rendimiento y la salud de los deportistas, pudiendo provocar diversas consecuencias a nivel personal y social, así como desarrollo de patologías crónicas a largo plazo e incluso abandono de la práctica deportiva (Hägglund et al., 2013; Lohmander et al., 2007; Ortín et al., 2010; Prien et al., 2020; Secrist et al., 2016). Por otro lado, las lesiones son también una carga financiera para los clubes de fútbol (López-Valenciano et al., 2020; Secrist et al., 2016).

No obstante, antes de aplicar estrategias preventivas y programas de intervención eficaces para reducir el riesgo de lesión, es preciso describir bien la magnitud, la gravedad y las principales características de las lesiones sufridas en el fútbol femenino (Hägglund et al., 2005; Van Mechelen et al., 1992; Webborn, 2012). Recientemente, un estudio ha combinado y meta-analizado la mayoría de los datos epidemiológicos disponibles en el fútbol femenino de élite y ha informado de una tasa de incidencia global de 6,1 lesiones por 1000 horas de exposición (López-Valenciano et al., 2021), que es ligeramente inferior a la documentada en fútbol masculino (8,1 lesiones por 1000 horas de exposición) (López-Valenciano et al., 2020). Según este meta-análisis, en el fútbol femenino la extremidad inferior presenta la tasa de incidencia más alta (4,8 lesiones/1000 h de exposición) y los tipos más comunes de lesiones son las músculo-tendinosas (principalmente de cuádriceps, isquiosurales y tríceps sural) (1,8 lesiones/1000 h de exposición), así como las articulares (no óseas) y ligamentosas (principalmente en el ligamento cruzado anterior de la rodilla y ligamentos laterales del tobillo) (1,5 lesiones/1000 h de exposición), que se asociaron con frecuencia a incidentes traumáticos sin contacto (LópezValenciano et al., 2021).

Si se analizan los estudios epidemiológicos llevados a cabo en fútbol femenino en particular, se observa que el principal foco de atención se localiza en el área de la rodilla, centrándose en la rotura de ligamento cruzado anterior (LCA) (Montalvo et al., 2018), que a su vez, ha sido destacada como una zona donde las lesiones se producen mayoritariamente sin contacto en futbolistas españolas (Del Coso et al., 2018). Además, los resultados de diferentes estudios epidemiológicos muestran que la incidencia de la lesión del LCA es de 2 a 4 veces mayor en mujeres que en hombres futbolistas (Montalvo et al., 2018; Pangrazio \& Forriol, 2016).

Por ello, conocer los factores de riesgo de lesión, en especial los factores de riesgo de lesiones ligamentosas de rodilla como la del LCA, podría ayudar a generar mejores estrategias de evaluación del riesgo lesional para la posterior intervención con mujeres futbolistas (Faude et al., 2006).

Shimokochi \& Shultz (2008) afirman que las lesiones del LCA suelen producirse cuando un individuo intenta desacelerar el cuerpo durante el aterrizaje tras un salto o durante la carrera mientras la rodilla está en un ángulo de flexión poco profundo. En este sentido, es preciso señalar que en el fútbol, un porcentaje elevado de roturas del LCA se producen sin contacto debido a la desaceleración brusca con la rodilla bloqueada en extensión, en tareas de cambio de dirección o al caer después de un salto (Kaneko et al., 2017; Villa et al., 2020; Waldén 
et al., 2015). Por tanto, el análisis biomecánico de mecánicas de aterrizaje o de cambio de dirección resulta especialmente relevante para la evaluación del riesgo de lesión del LCA en deporte femenino y especialmente en fútbol (Della Villa et al., 2021; Hewett et al., 2005; Myer et al., 2008; Robles-Palazón et al., 2021; Weir et al., 2019).

Las estrategias de corte o de cambio de dirección según Dos'Santos, McBurnie, et al. (2019), se dividen en cuatro fases consecutivas: aceleración inicial (aceleración positiva), desaceleración preliminar (aceleración negativa: para reducir el impulso en el COD sobre el penúltimo contacto y los pasos anteriores), corte/COD (aceptación del peso durante el último apoyo y empuje o impulsión que lleva al cambio en la dirección del movimiento) y, finalmente, reaceleración en la nueva dirección. Además, en el estudio diferencian tres tipos de corte: paso lateral $(\mathrm{PL})$ o side-step, corte cruzado (CC) o crossover y paso dividido (PD) o split-step.

Se considera una estrategia de paso lateral (PL) cuando un deportista planta su pie lateralmente respecto a la nueva dirección prevista. El cuerpo suele girar hacia la dirección de desplazamiento prevista, y el deportista acelera hacia la dirección opuesta a la pierna plantada con la que se realiza el último apoyo (Dos'Santos, McBurnie, Thomas, et al., 2019).

Es importante tener en cuenta la carga de la articulación de la rodilla y el riesgo de lesión según las estrategias de cambio de dirección. Comparando el $\mathrm{PL}$ frente al CC, algunos autores han observado que el primero provoca flexión de rodilla, valgo y rotación interna mientras que el segundo provoca flexión de rodilla, varo y rotación externa (Dos'Santos, McBurnie, Thomas, et al., 2019). Este hecho indicaba que los $\mathrm{PL}$ provocan mayor carga en la articulación de la rodilla, lo que puede aumentar la tensión del LCA y el ligamento colateral medial, aumentado con ello el riesgo de lesión (Dos'Santos, McBurnie, Thomas, et al., 2019).

Es interesante mencionar que varios estudios también han informado de posturas y mecánicas de mayor riesgo durante los $\mathrm{PL}$ en comparación con los CC, como mayores momentos abductores de la cadera, mayor valgo de la rodilla, mayor rotación interna de la cadera y ángulos de abducción, así como menor flexión de la cadera y la rodilla. Estos datos son preocupantes ya que estas cinemáticas aparecen en estudios de observación con respecto a las lesiones del LCA sin contacto y son posturas asociadas con una mayor carga de la articulación de la rodilla (Dos'Santos, McBurnie, Thomas, et al., 2019).

No obstante, son pocos los estudios que han evaluado la mecánica del COD para analizar el riesgo de lesión del LCA en el deporte y muy pocos los que han aplicado procedimientos e instrumentos válidos, fiables y de campo para dicha evaluación (Fox et al., 2016; Marques et al., 2020). Recientemente, se han desarrollado algunas propuestas para el análisis cinemático del COD en un contexto de campo, como la herramienta de observación cualitativa para el análisis cinemático de un $\mathrm{PL}$ de $90^{\circ}$ denominada "Cutting Movement Assessment Score" (CMAS) (Dos'Santos, McBurnie, Donelon, et al., 2019; Jones et al., 2017), o el método para el análisis biomecánico bidimensional de variables cinemáticas cuantitativas en una maniobra de COD descrito por Weir et al. (2019).

La evaluación del cambio de dirección podría contribuir de forma importante tanto a orientar el entrenamiento funcional preventivo como a guiar los procesos de recuperación de las futbolistas que ya han sufrido una rotura del LCA (Dos'Santos, Thomas, Comfort et al., 2019b; Marques et al., 2020). Sin embargo, ninguna de las herramientas de evaluación propuestas ha sido aplicada aún con jugadoras de fútbol $y$, por lo tanto, hasta el momento no existen valores de referencia que puedan indicar las características biomecánicas más comunes de las mujeres futbolistas al realizar un cambio de dirección ( $\mathrm{PL}$ ). Además, se desconoce en qué medida la manifestación de mecánicas de riesgo en un COD podría afectar el nivel de rendimiento de las futbolistas (Dos'Santos et al., 2017; Fox, 2018).

Por todo ello, los objetivos del presente estudio fueron describir la mecánica sagital del cambio de dirección de un grupo de jugadoras de fútbol y comparar la mecánica sagital de cambio de dirección, así como el rendimiento de las jugadoras en la prueba en función de su nivel competitivo. 


\section{Método}

Diseño de la investigación y procedimiento de evaluación del COD

Se diseñó un estudio observacional descriptivo de corte transversal en el cual se llevó a cabo una recogida de datos cuantitativos (tanto de rendimiento como cinemáticos) durante una prueba de cambio de dirección realizada con jóvenes jugadoras de fútbol.

En primer lugar, se realizó un calentamiento que consistió en carrera a baja intensidad, ejercicios de movilidad articular y ejercicios de activación neuromuscular (saltos, aceleraciones, desaceleraciones y cambios de dirección), con una duración total de 10 minutos aproximadamente (Dos'Santos et al., 2018). Previamente a la ejecución de la prueba, se registraron algunas variables antropométricas de las jugadoras como el peso, la altura y la longitud de ambas piernas.

Posteriormente, la prueba fue ejecutada por todas las futbolistas en un momento determinado durante su horario habitual de entrenamiento y en el propio campo de fútbol. La prueba consistió en una carrera de aproximación de $5 \mathrm{~m}$, seguida de la realización de un cambio de dirección de $90^{\circ}$ y la posterior reaceleración en la nueva dirección para realizar una carrera final de $3 \mathrm{~m}$ (figura 1) (Dos'Santos, McBurnie, Donelon, et al., 2019; Jones et al., 2017). Las jugadoras debían realizar al menos tres intentos válidos a cada lado.

Una repetición se consideró nula en los siguientes casos: a) Si se observaba que la jugadora no estaba realizando un esfuerzo máximo.

b) Cuando la jugadora pasaba por encima del cono o giraba antes del cono.

c) Cuando había algún problema técnico con las cámaras.

Cada repetición fue grabada para el posterior análisis de la mecánica sagital en el software kinovea (versión 8.15), mientras que el tiempo de ejecución fue registrado mediante células fotoeléctricas (Microgate Witty photocells, Italy). Para el análisis solamente se analizó una repetición a cada lado, que se escogió teniendo en cuenta una clara estrategia de paso lateral, bajo criterio de la observadora.

Siguiendo el procedimiento descrito por Weir et al. (2019), las variables cinemáticas analizadas desde el plano sagital fueron la flexión de tronco y la flexión de rodilla (figura 2). En cuanto a variables de rendimiento, se registraron tanto el tiempo de aproximación en la carrera de 3,5 m, como el tiempo total de ejecución en segundos.

\section{Participantes}

La muestra del estudio estuvo compuesta por 34 jugadoras de entre 16-28 años pertenecientes a dos equipos femeninos de diferente nivel competitivo. En concreto, participaron 13 jugadoras procedentes de un equipo amateur, que jugaba en una liga regional y entrenaba tres días por semana, y 21 jugadoras de un equipo de élite, que compite a nivel nacional y entrena cinco días por semana.

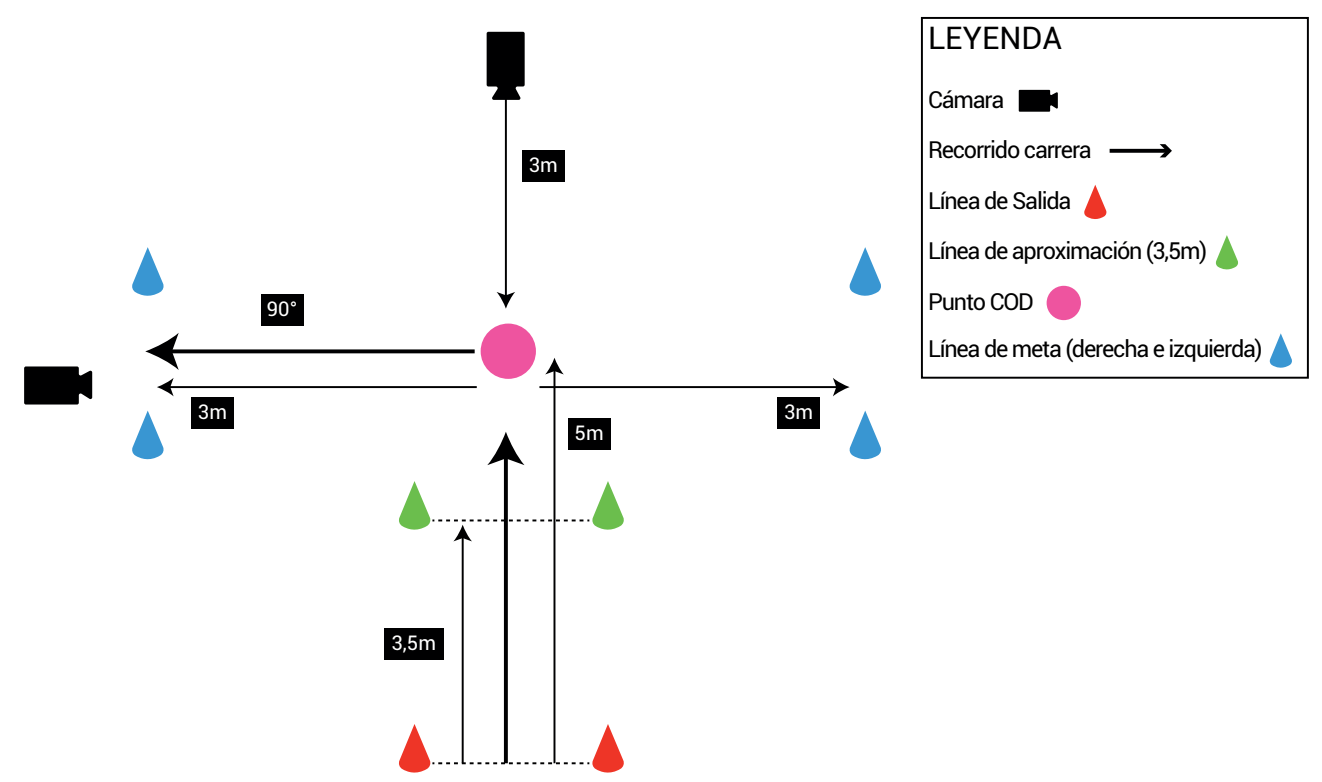

Figura 1. Esquema de la prueba de cambio de dirección de $90^{\circ}$ realizada por las jugadoras. 


\begin{tabular}{|c|c|c|}
\hline Ángulo & Flexión de tronco (FT) & Flexión de rodilla (FR) \\
\hline Líneas de referencia & $\begin{array}{l}\text { 1. Eje vertical trazado desde el GT } \\
\text { 2. Línea desde el GT hasta el ACR }\end{array}$ & $\begin{array}{l}\text { 1. Línea desde el GT hasta el LFC } \\
\text { 2. Línea desde el LFC hasta el LMAL }\end{array}$ \\
\hline $\begin{array}{l}\text { Medida registrada } \\
\text { (unidades) }\end{array}$ & $\begin{array}{c}\text { Ángulo formado entre la línea } 1 \text { y la línea } 2 \\
\text { (grados) }\end{array}$ & $\begin{array}{l}\text { Ángulo posterior formado entre la línea } 1 \text { y la línea } 2 \\
\text { (grados) } \\
\text { *Valor analizado: } 180^{\circ} \text { menos el valor angular registrado }\end{array}$ \\
\hline \multirow[t]{4}{*}{$\begin{array}{l}\text { Momentos de } \\
\text { evaluación/ registro }\end{array}$} & $\begin{array}{l}\text { A) Valor registrado durante el apoyo final: en el } \\
\text { momento del IC }\end{array}$ & $\begin{array}{l}\text { A) Valor registrado durante el penúltimo apoyo: en el } \\
\text { momento del IC }\end{array}$ \\
\hline & $\begin{array}{l}\text { B) Máximo valor encontrado durante el apoyo } \\
\text { final: desde el IC y a lo largo de la WA }\end{array}$ & \\
\hline & $\begin{array}{l}\text { C) Valor registrado durante el penúltimo } \\
\text { apoyo: en el momento del IC }\end{array}$ & $\begin{array}{l}\text { B) Máximo valor encontrado durante el penúltimo apoyo: } \\
\text { desde el IC y a lo largo de la WA }\end{array}$ \\
\hline & $\begin{array}{c}\text { D) Máximo valor encontrado durante el } \\
\text { penúltimo apoyo: desde el IC y a lo largo de } \\
\text { la WA }\end{array}$ & \\
\hline 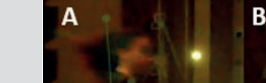 & 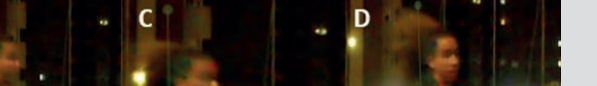 & A \\
\hline $\begin{array}{l}\text { Interpretación de } \\
\text { valores }\end{array}$ & $\begin{array}{l}(+) \text { : flexión } \\
(-) \text { : extensión }\end{array}$ & $\begin{array}{c}\text { (+): flexión } \\
\text { (-): hiper-extensión }\end{array}$ \\
\hline
\end{tabular}

Figura 2. Procedimiento de análisis de la cinemática bidimensional cuantitativa de un cambio de dirección en el plano sagital. GT: gran trocánter o trocánter mayor del fémur; ACR: acromion; LFC: cóndilo lateral del fémur; LMAL: maléolo lateral del tobillo; IC: contacto inicial o initial contact (foot strike); WA: fase de aceptación del peso o weight acceptance.

Lasjugadoras del equipo amateur presentaron

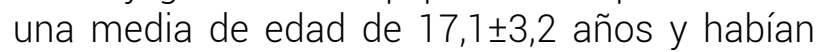
estado federadas en fútbol una media de 1,8 $\pm 1,5$ años, en comparación con las del equipo de élite que presentaron una edad media de $21,8 \pm 3,2$

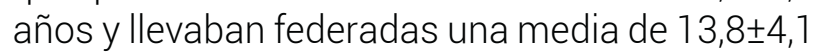
años.

El estudio se realizó siguiendo las directrices establecidas en la Declaración de Helsinki y el protocolo fue aprobado por el Comité de Ética e Investigación de la Universidad de Murcia (ID: 2424/2019). Por tanto, todas las jugadoras fueron informadas de los objetivos de la investigación y firmaron su consentimiento a participar voluntariamente en el estudio, pudiendo retirarse del mismo en cualquier momento.

\section{Análisis estadístico}

Los datos fueron tratados mediante estadística descriptiva e inferencial a través del programa "Statistical Package for Social Sciences" (IBM SPSS, versión 24.0). En primer lugar, se realizó un análisis descriptivo de todas las variables medidas que incluía medias, desviación estándar, máximos, mínimos, error estándar de la media, percentiles e intervalos de confianza al 95\% para cada una de las variables.

En segundo lugar, se llevó a cabo una comparación de medias en función del nivel competitivo de las jugadoras. Para analizar si las variables presentaban una distribución normal, se realizó la prueba Shapiro-Wilk y se determinó que tanto el tiempo de aproximación, como el ROM de flexión de tronco, la flexión de rodilla en el penúltimo apoyo y las variables de asimetría, presentaron una distribución no normal $(p<0,05)$. La comparación de medias entre los distintos grupos (jugadoras de élite vs. jugadoras amateur) se llevó a cabo mediante pruebas no paramétricas (prueba $U$ de Mann-Whitney para muestras independientes) para aquellas variables que presentaron una distribución no normal, mientras que se realizó mediante pruebas paramétricas (prueba $T$ para muestras independientes) para las variables que mostraron 
una distribución normal. Antes de realizar la prueba T para muestras independientes, se llevó a cabo la prueba de Levene para determinar la igualdad de varianzas entre grupos. Para todas las pruebas se estableció un nivel de significación basado en un valor de $p$ inferior a 0,05.

\section{Resultados}

En la tabla 1 se observan los resultados descriptivos de las variables analizadas en el cambio de dirección realizado por las 34 jugadoras participantes, tanto hacia la derecha como hacia la izquierda.

Respecto a las variables de rendimiento (tabla 1), cabe destacar que el tiempo de ejecución medio fue similar tanto hacia la derecha $(2,3 \pm 0,2 \mathrm{~s})$ como hacia la izquierda $(2,3 \pm 0,1 \mathrm{~s})$. El tiempo total de ejecución estuvo entre los 2,0 y los 2,6 segundos, aunque solamente el $25 \%$ de las jugadoras completaron la prueba en más de 2,4 segundos.

Como se muestra en la tabla 2, la media de flexión máxima de tronco en el apoyo final (DER: $12,8 \pm 7,7^{\circ} ; \mathrm{IZQ}: 11,1 \pm 7,1^{\circ}$ ) fue muy similar a la media de flexión de tronco en el contacto

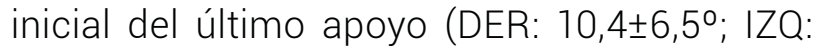
$\left.9,5 \pm 7,3^{\circ}\right)$.
Durante el penúltimo apoyo (PA), se encontró que la posición media del tronco fue en flexión en el momento del contacto inicial, tanto hacia la derecha $\left(7 \pm 4,8^{\circ}\right)$, como al realizar el COD hacia la izquierda $\left(6,1 \pm 5,6^{\circ}\right)$. Por otro lado, también se halló que la flexión de rodilla

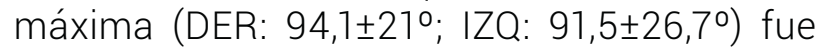
considerablemente superior (valores inferiores) a la flexión de rodilla en el contacto inicial (DER: 122,9 $\pm 21,1^{\circ} ;$ IZQ: $122,4 \pm 24,1^{\circ}$ ) durante el $\mathrm{PA}$, con un rango de flexión de rodilla media de $28,7 \pm 22,3^{\circ}$ al realizar el COD hacia la derecha, y de $30,9 \pm 29,7^{\circ}$, realizando el COD hacia la izquierda. Las mayores asimetrías en función de la dirección del COD, fueron encontradas en la flexión de rodilla, tanto inicial $\left(19,7 \pm 27,8^{\circ}\right)$ como máxima $\left(18 \pm 20,7^{\circ}\right)$.

En la tabla 3 se muestran el tiempo de ejecución y de aproximación, la media de cada una de las variables cinemáticas analizadas y las asimetrías medias encontradas en función del nivel competitivo de las futbolistas. En cuanto a variables de rendimiento, se halló que las jugadoras del equipo amateur realizaron el COD en un tiempo de aproximación y de ejecución significativamente mayor, tanto hacia la derecha como hacia la izquierda.

Tabla 1. Estadísticos descriptivos de las variables de rendimiento analizadas en el cambio de dirección para las 34 jugadoras de fútbol.

\begin{tabular}{|c|c|c|c|c|c|c|c|}
\hline \multicolumn{2}{|c|}{ Variable (unidad de medida) } & \multirow{2}{*}{$\frac{\text { Media } \pm \text { DE (IC 95\%) }}{2,3 \pm 0,2(2,3-2,4)}$} & \multirow{2}{*}{$\frac{\text { EEM }}{0}$} & \multirow{2}{*}{$\frac{\text { Percentil } \mathbf{2 5} \text { (IC 95\%) }}{2,2(2,2-2,3)}$} & \multirow{2}{*}{$\frac{\text { Percentil } 75 \text { (IC 95\%) }}{2,4(2,3-2,6)}$} & \multirow{2}{*}{$\frac{\text { Máx }}{2,6}$} & \multirow{2}{*}{$\frac{\text { Mín }}{2}$} \\
\hline Der & Tiempo ejecución (s) & & & & & & \\
\hline & Tiempo aproximación (s) & $1,1 \pm 0,1 \quad(1-1,1)$ & 0 & $1(0,9-1)$ & $1,1(1,1-1,2)$ & 1,4 & 0,9 \\
\hline \multirow[t]{2}{*}{$\mathrm{Izq}$} & Tiempo ejecución (s) & $2,3 \pm 0,1 \quad(2,3-2,4)$ & 0 & $2,2(2,2-2,3)$ & $2,4(2,3-2,5)$ & 2,6 & 2,1 \\
\hline & Tiempo aproximación (s) & $1 \pm 0,1 \quad(1-1,1)$ & 0 & $1(0,9-1)$ & $1,1(1,1-1,2)$ & 1,4 & 0,9 \\
\hline
\end{tabular}

IC 95\%: Intervalo de confianza al 95\%; DE: Desviación estándar; EEM: Error estándar de la media; Der: Cambio de dirección hacia la derecha; Izq: Cambio de dirección hacia la izquierda; Máx: Máximo; Mín: Mínimo.

Tabla 2. Estadísticos descriptivos de las variables cinemáticas analizadas en el cambio de dirección para las 34 jugadoras de fútbol.

\begin{tabular}{|c|c|c|c|c|c|c|c|}
\hline \multicolumn{2}{|c|}{ Variable (unidad de medida) } & \multirow{2}{*}{$\begin{array}{c}\begin{array}{c}\text { Media } \pm \text { DE } \\
\text { (IC 95\%) }\end{array} \\
10,4 \pm 6,5(8,1-12,7)\end{array}$} & \multirow{2}{*}{$\begin{array}{l}\text { EEM } \\
1,1\end{array}$} & \multirow{2}{*}{$\begin{array}{c}\begin{array}{c}\text { Percentil } 25 \\
\text { (IC 95\%) }\end{array} \\
5(4-8)\end{array}$} & \multirow{2}{*}{$\begin{array}{c}\begin{array}{c}\text { Percentil } 75 \\
\text { (IC 95\%) }\end{array} \\
15(12-18)\end{array}$} & \multirow{2}{*}{$\begin{array}{c}\text { Máx } \\
25\end{array}$} & \multirow{2}{*}{$\begin{array}{c}\text { Mín } \\
-2\end{array}$} \\
\hline Derecha & Flexión tronco en $\mathrm{Cl}\left({ }^{\circ}\right)$ & & & & & & \\
\hline & Flexión tronco máx $\left(^{\circ}\right)$ & $12,8 \pm 7,7(10,1-15,5)$ & 1,3 & $7(5-10)$ & $19(17-24)$ & 31 & -3 \\
\hline & Flexión tronco-ROM $\left(^{\circ}\right)$ & $2,4 \pm 3,9(1,1-3,8)$ & 0,7 & $0(0-2)$ & $4(2-7)$ & 18 & -1 \\
\hline & PA-Flexión tronco en $\mathrm{Cl}\left({ }^{\circ}\right)$ & $7 \pm 4,8(5,3-8,6)$ & 0,8 & $3(1-7)$ & $11(8-15)$ & 16 & -4 \\
\hline & PA-Flexión tronco máx $\left(^{\circ}\right)$ & $11,9 \pm 5,5(9,9-13,8)$ & 0,9 & $7(6-10)$ & $16(15-22)$ & 23 & 0 \\
\hline & PA-Flexión tronco-ROM $\left(^{\circ}\right)$ & $4,9 \pm 3,3(3,7-6)$ & 0,6 & $3(1-5)$ & $7(6-8)$ & 12 & 0 \\
\hline & PA-Flexión rodilla en $\mathrm{Cl}\left({ }^{\circ}\right)$ & $\begin{array}{c}122,9 \pm 21,1 \\
(115,5-130,2)\end{array}$ & 3,6 & $115(114-125)$ & $135(128-144)$ & 153 & 32 \\
\hline & PA-Flexión rodilla máx $\left(^{\circ}\right)$ & $94,1 \pm 21(86,8-101,4)$ & 3,6 & $88(79-94)$ & $107(103-111)$ & 118 & 30 \\
\hline & PA-Flexión rodilla-ROM $\left(^{\circ}\right)$ & $28,7 \pm 22,3(21-36,5)$ & 3,8 & $11(3-22)$ & $43(27-51)$ & 91 & 0 \\
\hline
\end{tabular}


Tabla 2. Estadísticos descriptivos de las variables cinemáticas analizadas en el cambio de dirección para las 34 jugadoras de fútbol (Continuación).

\begin{tabular}{|c|c|c|c|c|c|c|c|}
\hline \multicolumn{2}{|c|}{ Variable (unidad de medida) } & \multirow{2}{*}{$\begin{array}{c}\begin{array}{c}\text { Media } \pm \text { DE } \\
\text { (IC 95\%) }\end{array} \\
9,5 \pm 7,3(6,9-12)\end{array}$} & \multirow{2}{*}{$\begin{array}{c}\text { EEM } \\
1,3\end{array}$} & \multirow{2}{*}{$\begin{array}{c}\begin{array}{c}\text { Percentil 25 } \\
\text { (IC 95\%) }\end{array} \\
4(2-8)\end{array}$} & \multirow{2}{*}{$\begin{array}{c}\begin{array}{c}\text { Percentil } 75 \\
\text { (IC 95\%) }\end{array} \\
16(12-17)\end{array}$} & \multirow{2}{*}{\begin{tabular}{|c|} 
Máx \\
24
\end{tabular}} & \multirow{2}{*}{$\begin{array}{c}\text { Mín } \\
-5\end{array}$} \\
\hline Izquierda & Flexión tronco en $\mathrm{Cl}\left({ }^{\circ}\right)$ & & & & & & \\
\hline & Flexión tronco máx $\left(^{\circ}\right)$ & $11,1 \pm 7,1(8,6-13,6)$ & 1,2 & $5(3-9)$ & $17(16-19)$ & 25 & -3 \\
\hline & Flexión tronco-ROM $\left(^{\circ}\right)$ & $1,6 \pm 2,3(0,8-2,4)$ & 0,4 & $0(.-)$. & $2(2-5)$ & 10 & 0 \\
\hline & PA-Flexión tronco en $\mathrm{Cl}\left({ }^{\circ}\right)$ & $6,1 \pm 5,6(4,2-8,1)$ & 1 & $2(0-5)$ & $10(8-14)$ & 16 & -7 \\
\hline & PA-Flexión tronco máx $\left(^{\circ}\right)$ & $9,4 \pm 5,5(7,5-11,4)$ & 1 & $5(3-9)$ & $14(12-17)$ & 19 & -1 \\
\hline & PA-Flexión tronco-ROM $\left(^{\circ}\right)$ & $3,3 \pm 4,1(1,9-4,7)$ & 0,7 & $0(.-)$. & $5(3-9)$ & 17 & 0 \\
\hline & PA-Flexión rodilla en $\mathrm{Cl}\left(^{\circ}\right)$ & $122,4 \pm 24,1(114-130,9)$ & 4,1 & $115(115-126)$ & $134(129-146)$ & 155 & 13 \\
\hline & PA-Flexión rodilla máx $\left(^{\circ}\right)$ & $91,5 \pm 26,7(82,2-100,8)$ & 4,6 & $87(61-99)$ & $107(104-111)$ & 115 & 13 \\
\hline & PA-Flexión rodilla-ROM $\left(^{\circ}\right)$ & $30,9 \pm 29,7(20,6-41,3)$ & 5,1 & $9(0-20)$ & $41(32-61)$ & 119 & 0 \\
\hline \multirow{6}{*}{$\begin{array}{l}\text { Asi- } \\
\text { metrías }\end{array}$} & Flexión tronco en $\mathrm{Cl}\left({ }^{\circ}\right)$ & $6,4 \pm 5,4(4,6-8,3)$ & 0,9 & $3(1-5)$ & $9(6-11)$ & 25 & 0 \\
\hline & Flexión tronco máx $\left(^{\circ}\right)$ & $6,5 \pm 4,8(4,8-8,2)$ & 0,8 & $3(1-5)$ & $8(8-11)$ & 24 & 0 \\
\hline & PA-Flexión tronco en $\mathrm{Cl}\left({ }^{\circ}\right)$ & $5,1 \pm 4,4(3,5-6,6)$ & 0,8 & $2(1-4)$ & $7(5-9)$ & 19 & 0 \\
\hline & PA-Flexión tronco máx $\left(^{\circ}\right)$ & $5,6 \pm 4(4,2-7)$ & 0,7 & $3(2-6)$ & $7(6-10)$ & 20 & 0 \\
\hline & PA-Flexión rodilla en $\mathrm{Cl}\left({ }^{\circ}\right)$ & $19,7 \pm 27,8(10-29,4)$ & 4,8 & $6(4-10)$ & $23(16-32)$ & 140 & 1 \\
\hline & PA-Flexión rodilla máx $\left(^{\circ}\right)$ & $18 \pm 20,7(10,8-25,3)$ & 3,6 & $4(2-8)$ & $25(14-35)$ & 81 & 0 \\
\hline
\end{tabular}

PA: Penúltimo apoyo; Cl: Contacto inicial; IC 95\%: Intervalo de confianza al 95\%; DE: Desviación estándar; EEM: Error estándar de la media; ROM: Rango de movimiento; Der: Cambio de dirección hacia la derecha; Izq: Cambio de dirección hacia la izquierda; Máx: Máximo; Mín: Mínimo.

Tabla 3. Variables de rendimiento de las jugadoras de fútbol en un cambio de dirección según el nivel competitivo (amateur vs. Élite).

\begin{tabular}{|c|c|c|c|c|c|c|c|c|}
\hline \multicolumn{2}{|c|}{ Variables (unidad) } & \multicolumn{3}{|c|}{ Equipo amateur $(n=13)$} & \multicolumn{3}{|c|}{ Equipo de élite $(n=21)$} & \multirow{2}{*}{$\begin{array}{c}\mathrm{CM} \\
\text { Valor-p }\end{array}$} \\
\hline & & Media $\pm D E(E E M)$ & Máximo & Mínimo & Media $\pm D E(E E M)$ & Máximo & Mínimo & \\
\hline \multirow[t]{2}{*}{ Der } & $\mathrm{TE}(\mathrm{s})$ & $2,5 \pm 0,1(0)$ & 2,6 & 2,3 & $2,2 \pm 0,1(0)$ & 2,4 & 2 & $0,000+$ \\
\hline & $\mathrm{TA}(\mathrm{s})$ & $1,2 \pm 0,2(0)$ & 1,4 & 1 & $1 \pm 0,1(0)$ & 1,1 & 0,9 & $0,004 *$ \\
\hline \multirow[t]{2}{*}{ Izq } & $\mathrm{TE}(\mathrm{s})$ & $2,4 \pm 0,2(0)$ & 2,6 & 2,1 & $2,3 \pm 0,1(0)$ & 2,4 & 2,1 & $0,030 *$ \\
\hline & $\mathrm{TA}(\mathrm{s})$ & $1,1 \pm 0,1(0)$ & 1,4 & 0,9 & $1 \pm 0,1(0)$ & 1,2 & 0,9 & $0,003 *$ \\
\hline
\end{tabular}

Significación: * $<0,05 ;+p<0,001$.

DE: Desviación estándar; EEM: Error estándar de la media; Der: Cambio de dirección hacia la derecha; Izq: Cambio de dirección hacia la izquierda; CM: Comparación de medias; TE: Tiempo de ejecución; TA: Tiempo de aproximación; FT: Flexión del tronco; ROM: Rango de movimiento; FR: Flexión de rodilla; PA: Penúltimo apoyo; Cl: Contacto inicial.

Respecto a las variables cinemáticas (tabla 4), se encontró que el rango de movimiento medio de flexión de rodilla durante el penúltimo apoyo fue significativamente superior entre las jugadoras del equipo amateur $\left(43,5 \pm 32,5^{\circ}\right.$ vs. $23,2 \pm 25,6^{\circ}$, al realizar el COD hacia la izquierda. No obstante, el ROM medio de flexión de rodilla durante el PA al realizar el COD hacia la derecha fue más elevado en el equipo de élite (26,5 \pm $27,8^{\circ}$ vs. $\left.30,1 \pm 18,7^{\circ}\right)$, aunque sin diferencias significativas $(p=0,445)$. En este sentido, cabe destacar que la asimetría media en la máxima flexión de rodilla encontrada durante el PA fue más elevada en el equipo amateur $\left(25,7 \pm 22,9^{\circ}\right)$ que en el de élite $\left(13,3 \pm 18,2^{\circ}\right)$, con tendencia a diferencias estadísticamente significativas $(p=0,058)$.
En cambio, tanto la flexión de tronco inicial como máxima durante el último apoyo, presentaron valores significativamente superiores en el equipo de futbolistas de élite (inicial: $12,1 \pm 6,7^{\circ}$ vs. 5,2 $\pm 6,3^{\circ}$; máxima: $13,4 \pm 6,8^{\circ}$ vs. $\left.7,3 \pm 6^{\circ}\right)$.

\section{Discusión}

El objetivo principal del presente estudio fue describir la mecánica sagital del cambio de dirección (COD) de un grupo de jugadoras de fútbol. Para ello, se analizaron las mecánicas de flexión de rodilla y de flexión de tronco de las futbolistas durante el penúltimo y último apoyo en un COD de $90^{\circ}$. 
Tabla 4. Variables de rendimiento y variables cinemáticas de las jugadoras de fútbol en un cambio de dirección según el nivel competitivo (amateur vs. élite).

\begin{tabular}{|c|c|c|c|c|c|c|c|c|}
\hline \multicolumn{2}{|c|}{ Variables (unidad) } & \multicolumn{3}{|c|}{ Equipo amateur $(n=13)$} & \multicolumn{3}{|c|}{ Equipo de élite $(n=21)$} & \multirow{2}{*}{$\frac{\mathrm{CM}}{\text { Valor-p }}$} \\
\hline & & Media $\pm D E(E E M)$ & Máximo & Mínimo & Media $\pm D E(E E M)$ & Máximo & Mínimo & \\
\hline \multirow[t]{9}{*}{ Derecha } & FT en $\mathrm{Cl}\left({ }^{\circ}\right)$ & $9,2 \pm 6,7(1,9)$ & 22 & -2 & $11,1 \pm 6,5(1,4)$ & 25 & 1 & 0,433 \\
\hline & FT máxima $\left(^{\circ}\right)$ & $11,6 \pm 8(2,2)$ & 23 & -3 & $13,6 \pm 7,6(1,7)$ & 31 & 3 & 0,481 \\
\hline & $\mathrm{FT}-\mathrm{ROM}\left({ }^{\circ}\right)$ & $2,4 \pm 3,2(0,9)$ & 9 & -1 & $2,5 \pm 4,3(0,9)$ & 18 & 0 & 0,694 \\
\hline & $\mathrm{PA}-\mathrm{FT}$ en $\mathrm{Cl}\left({ }^{\circ}\right)$ & $6,5 \pm 4,3(1,2)$ & 16 & 0 & $7,3 \pm 5,1(1,1)$ & 16 & -4 & 0,633 \\
\hline & PA-FT máxima $\left({ }^{\circ}\right)$ & $11,5 \pm 5,5(1,5)$ & 23 & 0 & $12 \pm 5,7(1,2)$ & 22 & 2 & 0,798 \\
\hline & PA-FT-ROM ( $\left.{ }^{\circ}\right)$ & $5,1 \pm 3,2(0,9)$ & 10 & 0 & $4,8 \pm 3,5(0,8)$ & 12 & 0 & 0,794 \\
\hline & $\mathrm{PA}-\mathrm{FR}$ en $\mathrm{Cl}\left({ }^{\circ}\right)$ & $116,2 \pm 30(8,3)$ & 153 & 32 & $127 \pm 12(2,6)$ & 148 & 113 & 0,271 \\
\hline & PA-FR máxima $\left({ }^{\circ}\right)$ & $89,8 \pm 28,9(8)$ & 118 & 30 & $96,8 \pm 14,5(3,2)$ & 117 & 52 & 0,873 \\
\hline & PA-FR-ROM $\left(^{\circ}\right)$ & $26,5 \pm 27,8(7,7)$ & 91 & 0 & $30,1 \pm 18,7(4,1)$ & 69 & 2 & 0,445 \\
\hline \multirow[t]{9}{*}{ Izquierda } & FT en $\mathrm{Cl}\left({ }^{\circ}\right)$ & $5,2 \pm 6,3(1,7)$ & 17 & -5 & $12,1 \pm 6,7(1,5)$ & 24 & 2 & $0,005 *$ \\
\hline & FT máxima $\left(^{\circ}\right)$ & $7,3 \pm 6(1,7)$ & 17 & -3 & $13,4 \pm 6,8(1,5)$ & 25 & 2 & $0,012 *$ \\
\hline & FT-ROM $\left(^{\circ}\right)$ & $2,2 \pm 7,8(0,5)$ & 5 & 0 & $1,3 \pm 2,5(0,5)$ & 10 & 0 & 0,053 \\
\hline & $\mathrm{PA}-\mathrm{FT}$ en $\mathrm{Cl}\left({ }^{\circ}\right)$ & $4,5 \pm 5,1(1,4)$ & 14 & -7 & $7,2 \pm 5,8(1,3)$ & 16 & -3 & 0,173 \\
\hline & PA-FT máxima $\left(^{\circ}\right)$ & $7,5 \pm 5,1(1,4)$ & 17 & -1 & $10,6 \pm 5,6(1,2)$ & 19 & 1 & 0,117 \\
\hline & PA-FT-ROM $\left(^{\circ}\right)$ & $3,1 \pm 3,5(1)$ & 11 & 0 & $3,4 \pm 4,5(1)$ & 17 & 0 & 0,884 \\
\hline & PA-FR en $\mathrm{Cl}\left({ }^{\circ}\right)$ & $122,2 \pm 36(10)$ & 154 & 13 & $122,6 \pm 13,5(2,9)$ & 155 & 102 & 0,177 \\
\hline & PA-FR máxima $\left({ }^{\circ}\right)$ & $78,7 \pm 33,4(9,3)$ & 111 & 13 & $99,4 \pm 18,4(4)$ & 115 & 29 & 0,053 \\
\hline & PA-FR-ROM $\left(^{\circ}\right)$ & $43,5 \pm 32,5(9)$ & 119 & 0 & $23,2 \pm 25,6(5,6)$ & 111 & 0 & $0,038 *$ \\
\hline \multirow[t]{6}{*}{ Asimetría } & $\mathrm{FT}$ en $\mathrm{Cl}\left({ }^{\circ}\right)$ & $6,1 \pm 6,5(1,8)$ & 25 & 0 & $6,7 \pm 4,7(1)$ & 18 & 0 & 0,423 \\
\hline & FT máxima $\left(^{\circ}\right)$ & $7,1 \pm 6,3(1,7)$ & 24 & 0 & $6,1 \pm 3,8(0,8)$ & 15 & 0 & 0,901 \\
\hline & PA-FT en $\mathrm{Cl}\left({ }^{\circ}\right)$ & $5,4 \pm 4,2(1,2)$ & 16 & 1 & $4,9 \pm 4,7(1)$ & 19 & 0 & 0,556 \\
\hline & PA-FT máxima $\left(^{\circ}\right)$ & $6,5 \pm 5,5(1,5)$ & 20 & 1 & $5 \pm 2,8(0,6)$ & 12 & 0 & 0,592 \\
\hline & PA-FR en $\mathrm{Cl}\left({ }^{\circ}\right)$ & $30,2 \pm 41,9(11,6)$ & 140 & 1 & $13,2 \pm 10,1(2,2)$ & 33 & 1 & 0,375 \\
\hline & PA-FR máxima $\left({ }^{\circ}\right)$ & $25,7 \pm 22,9(6,3)$ & 75 & 2 & $13,3 \pm 18,2(4)$ & 81 & 0 & 0,058 \\
\hline
\end{tabular}

Significación: *p<0,05; †p<0,001.

DE: Desviación estándar; EEM: Error estándar de la media; Der: Cambio de dirección hacia la derecha; Izq: Cambio de dirección hacia la izquierda; CM: Comparación de medias; TE: Tiempo de ejecución; TA: Tiempo de aproximación; FT: Flexión del tronco; ROM: Rango de movimiento; FR: Flexión de rodilla; PA: Penúltimo apoyo; Cl: Contacto inicial.

En primer lugar, se halló que la media de flexión máxima de tronco en el apoyo final fue muy similar a la media de flexión de tronco en el contacto inicial del último apoyo. Este hallazgo podría deberse a que la máxima flexión de tronco se suela producir con frecuencia en un momento muy próximo al contacto inicial del último apoyo. Este resultado sugiere la posibilidad de utilizar solamente una de las dos medidas para el análisis de la flexión de tronco durante el último apoyo en futuros estudios. En línea con el presente estudio, Weir et al. (2019) describieron la evaluación de la flexión del tronco tanto en el momento del contacto inicial, como al final de la fase de aceptación del peso durante el último apoyo. Sin embargo, Della Villa et al. (2021) plantearon la evaluación de todas las variables cinemáticas en el momento de máxima flexión de rodilla durante el apoyo final. Esta reducción a un solo momento de medida podría facilitar el proceso de videoanálisis así como la interpretación de resultados.

Similar a lo hallado en la presente investigación, en un estudio donde se realizó videoanálisis del mecanismo lesional del LCA en fútbol masculino (Villa et al., 2020) se determinó que durante el contacto inicial (Cl) previo a la lesión, el tronco se mantiene erguido $\left(0^{\circ}\right)$, obteniendo datos semejantes durante el contacto final donde el tronco también se mantuvo erguido $\left(0^{\circ}\right)$. No obstante, a diferencia del estudio de Villa et al. (2020), en el presente estudio se mostraron valores medios de flexión de tronco superiores a los $9^{\circ}$ en ambos casos. 
En este sentido, la estrategia de mayor flexión de tronco durante el último apoyo mostrada por las jugadoras del presente estudio podría considerarse de menor riesgo, pues como indicaron Hewett et al. (2009), las atletas que sufrieron lesión del LCA demostraron una menor flexión del tronco hacia delante que las mujeres del grupo control. En esta línea, Sasaki et al. (2015) concluyeron que un mayor ángulo del tronco en el contacto inicial se asoció a una posición más segura durante el aterrizaje y la deceleración con una sola pierna.

En relación al penúltimo apoyo, Jones et al. (2016) analizaron las diferencias cinemáticas y cinéticas entre el penúltimo contacto y el contacto final de un PL con 22 jugadoras de fútbol. Estos autores definen el contacto final como la fase durante un corte o pivote cuando un individuo hace contacto con el suelo e inicia el movimiento en una dirección diferente, mientras que se considera penúltimo contacto al apoyo que tiene lugar previamente al contacto final. Jones et al. (2016) sugieren que debe tenerse la capacidad de frenar para cambiar de dirección eficientemente, reduciendo así la carga absorbida por la rodilla durante el apoyo final y favoreciendo una estrategia de COD más segura, así como un mayor rendimiento.

Es evidente que el penúltimo contacto del pie juega un papel importante en la desaceleración al cambiar de dirección y por lo tanto, puede ser considerado como un «paso preparatorio». Las estrategias de frenado que enfatizan en el PA, podrían reducir la carga de la articulación de la rodilla durante el apoyo final, y por otro lado facilitar un mayor rendimiento (Dos'Santos, Thomas, Comfort, et al., 2019a).

Durante el PA, en el presente trabajo se encontró que la posición media del tronco fue en flexión en el momento del contacto inicial, tanto hacia la derecha como hacia la izquierda. Estos resultados difieren con los del estudio de Jones et al. (2016), que analizaron las características de la deceleración previa al COD en jugadoras de fútbol y hallaron que las futbolistas tendían a inclinarse hacia atrás o estaban más erguidas en todo momento para aumentar el frenado, mientras que durante el contacto final el tronco tendía a flexionarse hacia delante. No obstante, es de destacar que la flexión de tronco durante el PA en el presente estudio no fue muy elevada, encontrando valores de tan solo 6-7 grados de flexión.

Siguiendo los trabajos de Dos'Santos, McBurnie, et al. (2019) y Jones et al. (2017), para considerar una buena estrategia de deceleración en el último apoyo, debe observarse inclinación del tronco hacia atrás, un contacto adelantado del pie de apoyo respecto al centro de masas y un primer contacto del pie de apoyo realizado con el talón. En base a esta propuesta, se podría considerar que las jugadoras de fútbol que participaron en la presente investigación, no realizaron una estrategia de deceleración clara y eficiente.

En cuanto a la flexión de rodilla, es preciso señalar que la tibia presenta mayor potencial de trasladarse anteriormente en ángulos poco profundos de flexión de la rodilla (el desplazamiento es mayor a $30^{\circ}$ que a $90^{\circ}$ de flexión de la rodilla), ya que las contracciones del cuádriceps son capaces de producir distensiones del LCA entre los $0^{\circ}$ y $30^{\circ}$ de flexión de la rodilla (Quatman et al., 2010). La tensión máxima del LCA ocurre cuando los ángulos de flexión de rodilla son más bajos (Fox, 2018). De hecho, una postura más erguida de los miembros inferiores, con una menor flexión de cadera y flexión de rodilla reduce la capacidad de atenuar y absorber la carga en la rodilla (Fox, 2018). Por lo tanto, una mayor flexión de rodilla se considera una estrategia más eficiente para amortiguar la fuerza y reducir la carga sobre el LCA de la rodilla.

En el presente trabajo, se hallaron valores de flexión de rodilla máxima considerablemente superiores $\left(86-89^{\circ}\right)$ a los de flexión de rodilla en el contacto inicial $\left(57-58^{\circ}\right)$ durante el PA, con rangos de flexión de rodilla media de 29$31^{\circ}$. Tal como se observa, en todos los casos las jugadoras realizaron una flexión de rodilla por encima de los $30^{\circ}$ indicados en la literatura previa, por lo que los valores de flexión de rodilla del presente estudio podrían considerarse como óptimos para una reducida carga en la rodilla.

Della Villa et al. (2021) analizaron el COD de 34 futbolistas amateur y de élite (18 hombres y 16 mujeres), planteando como mecánica de mayor riesgo una flexión máxima de rodilla inferior a los $50^{\circ}$ y como mecánica de riesgo medio, una flexión de rodilla máxima entre 50-70. De acuerdo a estos valores de referencia, la flexión 
de rodilla de las futbolistas de este estudio podría ser calificada como óptima y eficiente desde una perspectiva de reducción del riesgo de lesión. De hecho, la flexión simultánea de la cadera, la rodilla (hasta $\sim 100^{\circ}$ ) y el tobillo para absorber la carga en el plano sagital, facilita la aplicación de la fuerza de frenado durante más tiempo y, por lo tanto, favorece la reducción de la carga en la rodilla (Dos'Santos, Thomas, Comfort, et al., 2019a).

Sin embargo, hay que señalar que en el estudio de Della Villa et al. (2021), la flexión de rodilla fue analizada durante el último apoyo previo al COD, lo que dificulta la comparación de resultados con el presente estudio, donde únicamente se analizó la flexión en el penúltimo apoyo, al no disponer de imágenes óptimas de la flexión de rodilla en el último apoyo.

Un segundo objetivo fue comparar la mecánica sagital de cambio de dirección, así como el rendimiento de las jugadoras en la prueba en función de su nivel competitivo.

Tanto la flexión de tronco inicial como máxima durante el último apoyo, presentaron valores significativamente superiores en el equipo de futbolistas de élite. En esta línea, se ha demostrado que una estrategia biomecánica adecuada para reducir el riesgo de lesión es realizar una flexión y rotación del tronco hacia la nueva dirección prevista (Fox, 2018). Por lo tanto, las futbolistas de élite mostraron una mecánica del tronco de menor riesgo durante el último apoyo.

Por el contrario, el rango de movimiento medio de flexión de rodilla durante el penúltimo apoyo fue significativamente superior entre las jugadoras del equipo amateur $\left(43,5 \pm 32,5^{\circ}\right.$ vs. $\left.23,2 \pm 25,6^{\circ}\right)$, al realizar el COD hacia la izquierda. Es preciso señalar que mayores valores de flexión de rodilla podrían asociarse a un mayor tiempo de contacto durante el apoyo, y por lo tanto, podrían relacionarse con tiempos de ejecución mayores. En el estudio de Fox (2018) demuestran que se requiere un menor tiempo de contacto con el suelo para realizar el COD más rápido, a fin de invertir menos tiempo absorbiendo fuerza e impulsándose en la nueva dirección. En este sentido, cabe destacar que las jugadoras del equipo amateur fueron significativamente más lentas en la ejecución del COD que las jugadoras del equipo de élite, tanto hacia la derecha como hacia la izquierda. Los PL realizados a mayor velocidad de aproximación y con un mayor ángulo de corte requieren una mayor desaceleración y absorción de la carga para realizar el movimiento. Cuando se aumenta la velocidad, aumenta la carga en la rodilla y con ello, el riesgo de lesión (Fox, 2018). Sin embargo, es posible que las jugadoras de élite realizaran menor flexión de rodilla como estrategia para aumentar su rendimiento en el COD, a pesar de que esto supusiera una mayor carga para la rodilla. Estos resultados contrastan con los del estudio de Sigward \& Powers (2006), quienes hallaron que eran los atletas novatos los que presentaban menor flexión de rodilla frente a los experimentados.

Fox (2018) sugiere aumentar la flexión de rodilla para mejorar la mecánica del COD, sin embargo, plantea el dilema de que esta mejora a nivel biomecánico podría ser perjudicial para el rendimiento ya que, reduciría la velocidad de ejecución del cambio de dirección.

El mayor rendimiento de las jugadoras de élite en la ejecución de la prueba, podría deberse a una mayor capacidad de las mismas en cuanto a fuerza excéntrica de miembros inferiores. En relación a este resultado, Jones et al. (2017) determinaron que las jugadores de fútbol con mayor fuerza excéntrica fueron las que realizaron más rápido el COD, sobre todo aquellas con mayor fuerza excéntrica de extensión de rodilla, ya que eran capaces de desacelerar durante el penúltimo apoyo partiendo desde una velocidad de aproximación más rápida. En este estudio, las jugadoras más fuertes fueron las que registraron velocidades de aproximación más rápidas y una mayor reducción de la velocidad durante el penúltimo apoyo en comparación con las más débiles.

Por otro lado, los resultados del presente trabajo mostraron que las mayores asimetrías en función de la dirección del COD, fueron encontradas en la flexión de rodilla, tanto inicial $\left(20^{\circ}\right)$ como máxima $\left(18^{\circ}\right)$. Para reducir esos desequilibrios, se ha aconsejado realizar entrenamientos con mayor carga de ejercicios unilaterales respecto a ejercicios bilaterales (Bishop et al., 2019).

Así pues, la asimetría media en la máxima flexión de rodilla encontrada durante el PA fue más elevada en el equipo amateur $\left(25,7 \pm 22,9^{\circ}\right)$ 
que en el de élite $\left(13,3 \pm 18,2^{\circ}\right)$. McLean et al. (1999) afirmaron que el nivel de experiencia fue el único factor que tenía un efecto significativo en la variabilidad cinemática de la articulación de la rodilla, poniendo de manifiesto que la variabilidad extrema en diferentes repeticiones de una misma prueba o tarea global, se ha asociado comúnmente con bajos niveles de experiencia o preparación física. En este sentido, aunque no se trató de diferencias significativas, es probable que las jugadoras amateurs mostraran mayores asimetrías debido a una menor consistencia en su ejecución de la técnica del COD.

\section{Conclusiones} fueron:

Las conclusiones de la presente investigación

- La media de flexión máxima de tronco en el apoyo final fue muy similar a la media de flexión de tronco en el contacto inicial del último apoyo, por lo que la reducción a un solo momento de medida podría facilitar el proceso de videoanálisis así como la interpretación de resultados en futuros estudios.

- Las jugadoras de fútbol mostraron una estrategia de flexión de tronco de bajo riesgo durante el apoyo final, con valores medios de flexión de tronco superiores a los $9^{\circ}$ tanto en el contacto inicial como en la máxima flexión del último apoyo.

- Las jugadoras de fútbol que participaron en la presente investigación no realizaron una estrategia de deceleración clara y eficiente, ya que durante el penúltimo apoyo, las futbolistas presentaron una posición media de flexión del tronco. Sin embargo, sí que presentaron una adecuada flexión de rodilla durante el penúltimo apoyo.

- Las futbolistas de élite realizaron el cambio de dirección más rápido y de forma más segura, presentando una mayor flexión del tronco durante el último apoyo. Sin embargo, realizaron una menor flexión de rodilla durante el penúltimo apoyo que las jugadoras del equipo amateur.

- Las mayores asimetrías en función del sentido del cambio de dirección, se dieron en cuanto a flexión de rodilla, encontrando los mayores valores de asimetrías en el equipo de nivel amateur.

\section{Aplicaciones prácticas}

El presente trabajo constituye un ejemplo de valoración del riesgo de lesión mediante la evaluación de la mecánica del cambio de dirección con jóvenes jugadoras de fútbol en un contexto práctico real. De esta manera, los procedimientos descritos podrán servir de guía a los/las profesionales de las Ciencias del Deporte para la aplicación de la prueba y el análisis de la mecánica de cambio de dirección con sus propios deportistas. En este sentido, los datos biomecánicos aportados en el presente estudio con jugadoras de fútbol, podrán ser utilizados como referencia para la interpretación de los valores registrados en otros equipos de similares características.

En concreto, se destaca la recomendación de realizar el análisis cinemático de la flexión del tronco únicamente en el momento de máxima flexión durante el último apoyo, ya que los valores encontrados en este momento fueron muy similares a los hallados en el contacto inicial del apoyo final. Por otra parte, se ha de tener en consideración que los datos extraídos de la evaluación biomecánica del cambio de dirección podrían contribuir de forma importante tanto a orientar el entrenamiento funcional preventivo como a guiar los procesos de recuperación de las futbolistas que ya han sufrido una rotura del LCA. En este sentido, dado que las jugadoras de fútbol que participaron en la presente investigación no realizaron una estrategia de deceleración clara y eficiente, sería recomendable el entrenamiento específico de la mecánica de deceleración en el penúltimo apoyo a nivel general con todas las futbolistas. En base a los resultados del presente trabajo, ha de tenerse en cuenta también, que las jugadoras con menos experiencia deberían hacer mayor hincapié en equilibrar su ejecución mecánica tanto hacia la derecha como hacia la izquierda mediante entrenamiento compensatorio para reducir las asimetrías. Además, dado que las futbolistas de élite realizaron el cambio de dirección más rápido y de forma más segura, cabe destacar que el entrenamiento específico de la mecánica del cambio de dirección podría resultar especialmente relevante para reducir el riesgo de lesión de 
las futbolistas con menor experiencia o nivel competitivo.

La falta de valoración de la flexión de rodilla en el último apoyo, constituye una de las principales limitaciones del presente trabajo, no obstante, este análisis no fue posible debido a la grabación de la prueba desde una perspectiva sagital que impedía la visualización del ángulo de flexión de rodilla en el apoyo final del COD. Por lo tanto, se recomienda la grabación de la prueba desde tres puntos: desde ambas perspectivas en el plano sagital, así como desde una vista frontal. De igual forma, futuros estudios deberían analizar la cinemática del cambio de dirección con una muestra más amplia de jugadoras de fútbol de distinto nivel competitivo y contemplando un mayor número de variables cuantitativas, tanto en el plano sagital (relativas a las articulaciones de cadera y tobillo) como en el plano frontal.

\section{Financiación}

Este estudio es parte del Proyecto de I+D+i/ PID2020-115886RB-I00 "El Fútbol Femenino Importa: Identificación del Riesgo de Lesión a través de la Inteligencia Artificial" financiado por MCIN/AEI/10.13039/501100011033

\section{Agradecimientos}

La investigadora Alba Aparicio Sarmiento ha participado en el presente trabajo gracias a la Ayuda del programa de Formación de Profesorado Universitario del Ministerio de Ciencia, Innovación y Universidades (Referencia: FPU18/00702).

\section{Referencias}

Alahmad, T. A., Kearney, P., \& Cahalan, R. (2020). Injury in elite women's soccer: A systematic review. The Physician and Sportsmedicine, $0(0), 1-7$.

https://doi.org/10.1080/00913847.2020.1720548

Aliendre Morel, C. R., \& Contrera, M. (2019). La discriminación de género en el deporte. El caso del futbol femenino. ScientiAmericana, 6(2), 81-90.

Bishop, C., Turner, A., Maloney, S., Lake, J., Loturco, I., Bromley, T., \& Read, P. (2019). Drop Jump Asymmetry is Associated with Reduced Sprint and Change-of-Direction Speed Performance in Adult Female Soccer Players. Sports, 7(1), 29. https://doi.org/10.3390/sports7010029

Bradley, P., \& Scott, D. (2019). Análisis físico de la Copa Mundial Femenina de la FIFA Francia 2019 (p. 169).
Fédération Internationale de Football Association. https:// digitalhub.fifa.com/m/62485258e710dacf/original/ pwinph2nr4snbh5u2wsq-pdf.pdf

Cardoso de Araújo, M. de, \& Mießen, K. A. M. (2017). Twenty Years of the FIFA Women's World Cup: An Outstanding Evolution of Competitiveness. Women in Sport and Physical Activity Journal 25(1), 60-64. https://doi.org/10.1123/wspaj.2015-0047

Del Coso, J., Herrero, H., \& Salinero, J. J. (2018). Injuries in Spanish female soccer players. Journal of Sport and Health Science, 7(2), 183-190. https://doi.org/10.1016/j.jshs.2016.09.002

Della Villa, F., Di Paolo, S., Santagati, D., Della Croce, E., Lopomo, N. F., Grassi, A., \& Zaffagnini, S. (2021). A 2D video-analysis scoring system of $90^{\circ}$ change of direction technique identifies football players with high knee abduction moment. Knee Surgery, Sports Traumatology, Arthroscopy. https://doi.org/10.1007/s00167-021-06571-2

Dos'Santos, T., McBurnie, A., Donelon, T., Thomas, C., Comfort, P. \& Jones, P. A. (2019). A qualitative screening tool to identify athletes with «high-risk " movement mechanics during cutting: The cutting movement assessment score (CMAS). Physical Therapy in Sport, 38, 152-161.

https://doi.org/10.1016/j.ptsp.2019.05.004

Dos'Santos, T., McBurnie, A., Thomas, C., Comfort, P., \& Jones, P. A. (2019). Biomechanical Comparison of Cutting Techniques: A Review and Practical Applications. Strength \& Conditioning Journal, 41(4), 40-54. https://doi.org/10.1519/SSC.0000000000000461

Dos'Santos, T., Thomas, C., Comfort, P., \& Jones, P. A. (2019a). Role of the Penultimate Foot Contact During Change of Direction: Implications on Performance and Risk of Injury. Strength \& Conditioning Journal, 41(1), 87-104 https://doi.org/10.1519/SSC.0000000000000395

Dos'Santos, T., Thomas, C., Comfort, P., \& Jones, P. A. (2019b). The Effect of Training Interventions on Change of Direction Biomechanics Associated with Increased Anterior Cruciate Ligament Loading: A Scoping Review. Sports Medicine, 49(12), 1837-1859. https://doi.org/10.1007/s40279-019-01171-0

Dos'Santos, T., Thomas, C., Jones, P. A., \& Comfort, P. (2017). Mechanical Determinants of Faster Change of Direction Speed Performance in Male Athletes. Journal of Strength and Conditioning Research, 31(3), 696-705. https://doi.org/10.1519/JSC.0000000000001535

Faude, O., Junge, A., Kindermann, W., \& Dvorak, J. (2006). Risk factors for injuries in elite female soccer players. British Journal of Sports Medicine, 40(9), 785-790. https://doi.org/10.1136/bjsm.2006.027540

Federation Internationale de Football Association (2019). Women's Football MA's Survey Report (p. 113). http:// ac.els-cdn.com/S002175571500008X/1-s2.0S002175571500008X-main.pdf?_tid=68fff318-30ef11 e 7 - afa $2-00000$ a a cb3 5f\&acdnat $=1493919227$. eca904234cd6239317e02f316d6a2967

Figueres, E., Belloch, S., \& Perez-Soriano, P. (2008). Poster: Estudio epidemiológico en el fútbol.

Fox, A. S. (2018). Change-of-Direction Biomechanics: Is What's Best for Anterior Cruciate Ligament Injury Prevention Also Best for Performance? Sports Medicine, 48(8), 1799-1807. https://doi.org/10.1007/s40279-018-0931-3

Fuller, C. W., Ekstrand, J., Junge, A., Andersen, T. E., Bahr, R., Dvorak, J., Hagglund, M., McCrory, P., \& Meeuwisse, W. H. (2006). Consensus statement on injury definitions and data collection procedures in studies of football (soccer) injuries. Scandinavian Journal of Medicine and Science in Sports, 16(2), 83-92. https://doi.org/10.1111/j.1600-0838.2006.00528.x

Giza, E., Mithöfer, K., Farrell, L., Zarins, B., \& Gill, T. (2005). Injuries in women's professional soccer. British Journal of Sports Medicine, 39(4), 212-216. https://doi.org/10.1136/bjsm.2004.011973

Hägglund, M., Waldén, M., Bahr, R., \& Ekstrand, J. (2005). Methods for epidemiological study of injuries to professional football 
players: Developing the UEFA model. British Journal of Sports Medicine, 39(6), 340-346

https://doi.org/10.1136/bjsm.2005.018267

Hägglund, M., Waldén, M., Magnusson, H., Kristenson, K. Bengtsson, H., \& Ekstrand, J. (2013). Injuries affect team performance negatively in professional football: An 11-year follow-up of the UEFA Champions League injury study. British Journal of Sports Medicine, 47(12), 738-742.

https://doi.org/10.1136/bjsports-2013-092215

Hewett, T. E., Torg, J. S., \& Boden, B. P. (2009). Video analysis of trunk and knee motion during non-contact anterior cruciate ligament injury in female athletes: Lateral trunk and knee abduction motion are combined components of the injury mechanism. British Journal of Sports Medicine, 43(6), 417422. https://doi.org/10.1136/bjsm.2009.059162

Hewett, Timothy E., Myer, G. D., Ford, K. R., Heidt, R. S., Colosimo, A. J., McLean, S. G., van den Bogert, A. J., Paterno, M. V., \& Succop, P. (2005). Biomechanical Measures of Neuromuscular Control and Valgus Loading of the Knee Predict Anterior Cruciate Ligament Injury Risk in Female Athletes: A Prospective Study. The American Journal of Sports Medicine, 33(4), 492-501. https://doi.org/10.1177/0363546504269591

Jones, P. A., Herrington, L., \& Graham-Smith, P. (2016). Braking characteristics during cutting and pivoting in female soccer players. Journal of Electromyography and Kinesiology, 30, 4654. https://doi.org/10.1016/j.jelekin.2016.05.006

Jones, P. A., Thomas, C., Dos'Santos, T., McMahon, J. J., \& GrahamSmith, P. (2017). The Role of Eccentric Strength in $180^{\circ}$ Turns in Female Soccer Players. Sports, 5(2), 42. https://doi.org/10.3390/sports5020042

Jones, P., Donelon, T., \& Dos'Santos, T. (2017). A preliminary investigation into a qualitative assessment tool to identify athletes with high knee abduction moments during cutting: Cutting Movement Assessment Score (CMAS). Professional Strength \& Conditioning, 37-42.

Kaneko, S., Sasaki, S., Hirose, N., Nagano, Y., Fukano, M., \& Fukubayashi, T. (2017). Mechanism of Anterior Cruciate Ligament Injury in Female Soccer Players. Asian Journal of Sports Medicine, 8(1). https://doi.org/10.5812/asjsm.38205

Krustrup, P., \& Krustrup, B. R. (2018). Football is medicine: It is time for patients to play! British Journal of Sports Medicine, 52(22), 1412-1414. https://doi.org/10.1136/bjsports-2018-099377

Krustrup, P., Helge, E. W., Hansen, P. R., Aagaard, P., Hagman, M., Randers, M. B., de Sousa, M., \& Mohr, M. (2018). Effects of recreational football on women's fitness and health: Adaptations and mechanisms. European Journal of Applied Physiology, 118(1), 11-32.

https://doi.org/10.1007/s00421-017-3733-7

Llana, S., Soriano, P., \& Figueres, E. (2010). La epidemiología en el fútbol: Una revisión sistemática. Revista Internacional de Medicina y Ciencias de la Actividad Física y el Deporte, 10(37), 22-40. http://cdeporte.rediris.es/revista/revista37/ artfutbol130.htm

Lohmander, L. S., Englund, P. M., Dahl, L. L., \& Roos, E. M. (2007). The long-term consequence of anterior cruciate ligament and meniscus injuries: Osteoarthritis. The American Journal of Sports Medicine, 35(10), 1756-1769. https://doi.org/10.1177/0363546507307396

López-Valenciano, A., Raya-González, J., Garcia-Gómez, J. A., Aparicio-Sarmiento, A., Sainz de Baranda, P., De Ste Croix, M., \& Ayala, F. (2021). Injury Profile in Women's Football: A Systematic Review and Meta-Analysis. Sports Medicine, 1-20. https://doi.org/10.1007/s40279-020-01401-w

López-Valenciano, A., Ruiz-Pérez, I., Garcia-Gómez, A., Vera-Garcia, F. J., De Ste Croix, M., Myer, G. D., \& Ayala, F. (2020). Epidemiology of injuries in professional football: A systematic review and meta-analysis. British Journal of Sports Medicine, 54(12), 711718. https://doi.org/10.1136/bjsports-2018-099577

Marques, J. B., Paul, D. J., Graham-Smith, P., \& Read, P. J. (2020). Change of Direction Assessment Following Anterior Cruciate Ligament Reconstruction: A Review of Current Practice and Considerations to Enhance Practical Application. Sports
Medicine, 50(1), 55-72

https://doi.org/10.1007/s40279-019-01189-4

Mclean, S. G., Neal, R. J., Myers, P. T., \& Walters, M. R. (1999). Knee joint kinematics during the sidestep cutting maneuver: Potential for injury in women. Medicine and Science in Sports and Exercise, 31(7), 959-968. https://doi.org/10.1097/00005768-199907000-00007

Montalvo, A. M., Schneider, D. K., Silva, P. L., Yut, L., Webster, K. E. Riley, M. A., Kiefer, A. W., Doherty-Restrepo, J. L., \& Myer, G. D. (2019). 'What's my risk of sustaining an ACL injury while playing football (soccer)?' A systematic review with meta-analysis. British Journal of Sports Medicine, 53(21), 1333-1340.

Myer, G. D., Ford, K. R., \& Hewett, T. E. (2008). Tuck Jump Assessment for Reducing Anterior Cruciate Ligament Injury Risk. Athletic Therapy Today, 13(5), 39-44. https://www.ncbi.nlm.nih.gov/pmc/articles/PMC2779043/

Oja, P., Titze, S., Kokko, S., Kujala, U. M., Heinonen, A., Kelly, P., Koski, P., \& Foster, C. (2015). Health benefits of different sport disciplines for adults: Systematic review of observational and intervention studies with meta-analysis. British Journal of Sports Medicine, 49(7), 434-440.

https://doi.org/10.1136/bjsports-2014-093885

Ortín, F. J., Garcés de los Fayos, E. J., \& Olmedilla, A. (2010). Influencia de los factores psicológicos en las lesiones deportivas. Papeles del psicólogo, 31(3), 8.

Pangrazio, O., \& Forriol, F. (2016). Diferencias de las lesiones sufridas en 4campeonatos sudamericanos de fútbol femenino y masculino. Revista Latinoamericana de Cirugía Ortopédica, 1(2), 58-65. https://doi.org/10.1016/j.rslaot.2016.10.001

Peterson, L., Junge, A., Chomiak, J., Graf-Baumann, T., \& Dvorak, J. (2000). Incidence of Football Injuries and Complaints in Different Age Groups and Skill-Level Groups. The American Journal of Sports Medicine, 28(5_suppl), 51-57. https://doi.org/10.1177/28.suppl_5.s-51

Prien, A., Boudabous, S., Junge, A., Verhagen, E., Delattre, B. M. A., \& Tscholl, P. M. (2020). Every second retired elite female football player has MRI evidence of knee osteoarthritis before age 50 years: A cross-sectional study of clinical and MRI outcomes. Knee Surgery, Sports Traumatology, Arthroscopy, 28(2), 353362. Scopus. https://doi.org/10.1007/s00167-019-05560-w

Prieto Andreu, J. M. (2015). Variables deportivas y personales en la ocurrencia de lesiones deportivas. Diferencias entre deportes individuales y colectivos (Sport and personal variables in the occurrence of sports injuries. Differences between individual and team sports ). Retos, 28, 21-25. https://doi.org/10.47197/retos.v0i28.34819

Quatman, C. E., Quatman-Yates, C. C., \& Hewett, T. E. (2010). A «plane" explanation of anterior cruciate ligament injury mechanisms: A systematic review. Sports Medicine, 40(9), 729746. https://doi.org/10.2165/11534950-000000000-00000

Raya-González, J., \& Estévez-Rodríguez, J. (2016). Factores de riesgo asociados a la aparición de lesiones en el fútbol. Revista de Preparación física en el Fútbol, 21, 8-18.

Robles-Palazón, F., Ruiz-Pérez, I., Oliver, J., Ayala, F., \& Sainz de Baranda P. (2021). Reliability, validity, and maturation-related differences of frontal and sagittal plane landing kinematic measures during drop jump and tuck jump screening tests in male youth soccer players. Physical Therapy in Sport, 50, 206-216.

Sasaki, S., Nagano, Y., Kaneko, S., Imamura, S., Koabayshi, T., \& Fukubayashi, T. (2015). The Relationships Between the Center of Mass Position and the Trunk, Hip, and Knee Kinematics in the Sagittal Plane: A Pilot Study on Field-Based Video Analysis for Female Soccer Players. Journal of Human Kinetics, 45, 71 80. https://doi.org/10.1515/hukin-2015-0008

Secrist, E. S., Bhat, S. B., \& Dodson, C. C. (2016). The Financial and Professional Impact of Anterior Cruciate Ligament Injuries in National Football League Athletes. Orthopaedic Journal of Sports Medicine, 4(8) https://doi.org/10.1177/2325967116663921

Shimokochi, Y., \& Shultz, S. J. (2008). Mechanisms of Noncontact Anterior Cruciate Ligament Injury. Journal of Athletic Training, 43(4), 396-408. https://doi.org/10.4085/1062-6050-43.4.396 
Sigward, S., \& Powers, C. M. (2006). The influence of experience on knee mechanics during side-step cutting in females. Clinical Biomechanics, 21(7), 740-747.

https://doi.org/10.1016/j.clinbiomech.2006.03.003

UEFA. (2015). Women's Football across the National Associations 2014-15 (p. 93) [Análisis de la evolución del fútbol femenino entre las asociaciones nacionales de la UEFA]. Union of European Football Associations. Women`s football across the national associations (2014-15) (uefa.com)

UEFA.com. (16 de junio de 2010). Historia de la UEFA Champions League Femenina / UEFA Women's Champions League. UEFA. com. https://es.uefa.com/womenschampionsleague/ news/01 e6-0e1 1f212c6fe-8e4476f92fe1-1000--historia-dela-uefa-champions-league-femenina /

Valenti, M., Morrow, S., \& Scelles, N. (2018). Women's football studies: An integrative review. Sport, Business and Management: An International Journal, 8(5), 511-528. https://doi.org/10.1108/SBM-09-2017-0048

Van Mechelen, W., Hlobil, H., \& Kemper, H. C. (1992). Incidence, severity, aetiology and prevention of sports injuries. A review of concepts. Sports Medicine, 14(2), 82-99.

https://doi.org/10.2165/00007256-199214020-00002
Villa, F. D., Buckthorpe, M., Grassi, A., Nabiuzzi, A., Tosarelli, F., Zaffagnini, S., \& Villa, S. D. (2020). Systematic video analysis of ACL injuries in professional male football (soccer): Injury mechanisms, situational patterns and biomechanics study on 134 consecutive cases. British Journal of Sports Medicine, 54(23), 1423-1432. https://doi.org/10.1136/bjsports-2019-101247

Waldén, M., Krosshaug, T., Bjørneboe, J., Andersen, T. E., Faul, O., \& Hägglund, M. (2015). Three distinct mechanisms predominate in non-contact anterior cruciate ligament injuries in male professional football players: A systematic video analysis of 39 cases. British Journal of Sports Medicine, 49(22), 1452-1460. https://doi.org/10.1136/bjsports-2014-094573

Webborn, N. (2012). Lifetime injury prevention: The sport profile model. British Journal of Sports Medicine, 46(3), 193-197. https://doi.org/10.1136/bjsports-2011-090417

Weir, G., Alderson, J., Smailes, N., Elliott, B., \& Donnelly, C. (2019). A Reliable Video-based ACL Injury Screening Tool for Female Team Sport Athletes. International Journal of Sports Medicine, 40(3), 191-199. Scopus. https://doi.org/10.1055/a-0756-9659

Yanguas Leyes, J., Til Pérez, L., \& Cortés de Olano, C. (2011). Lesión del ligamento cruzado anterior en fútbol femenino. Estudio epidemiológico de tres temporadas. Apunts. Medicina de l'Esport, 46(171), 137-143. https://doi.org/10.1016/j.apunts.2011.02.006 11.02.006 